\title{
Clinical outcomes of bivalirudin versus heparin in atrial fibrillation patients undergoing percutaneous left atrial appendage occlusion
}

\author{
Xiaochun Zhang ${ }^{1 \#}$, Qinchun Jin ${ }^{1 \#}$, Dehong Kong ${ }^{2}$, Cuizhen Pan $^{2}$, Xian Zhang ${ }^{3}$, Dan Zhou ${ }^{3}$, Zhiyun Shen ${ }^{3}$, \\ Daxin Zhou ${ }^{1}$, Junbo Ge ${ }^{1}$ \\ ${ }^{1}$ Department of Cardiology, Zhongshan Hospital, Fudan University, Research Unit of Cardiovascular Techniques and Devices, Chinese Academy of Medical \\ Sciences, Shanghai, China; ${ }^{2}$ National Clinical Research Center for Interventional Medicine, Shanghai, China; ${ }^{3}$ Department of Cardiac Echocardiology, \\ Zhongshan Hospital, Fudan University, Shanghai, China; ${ }^{4}$ Department of Nursing, Zhongshan Hospital, Fudan University, Shanghai, China \\ Contributions: (I) Conception and design: X Zhang, Q Jin; (II) Administrative support: D Zhou, J Ge; (III) Provision of study materials or patients: D \\ Zhou, Z Shen; (IV) Collection and assembly of data: D Kong, C Pan; (V) Data analysis and interpretation: Q Jin, Z Shen; (VI) Manuscript writing: \\ All authors; (VII) Final approval of manuscript: All authors. \\ \#These authors contributed equally to this work. \\ Correspondence to: Daxin Zhou. Shanghai Institute of Cardiovascular Diseases, Department of Cardiology, Zhongshan Hospital, Fudan University, \\ 180 Fenglin Road, Shanghai 200032, China. Email: zhou.daxin@outlook.com.
}

Background: Prior studies have suggested that patients with atrial fibrillation (AF) referred for left atrial appendage occlusion (LAAO) are confronted with considerable risk of periprocedural thromboembolism and hemmorhagic events. The purpose of this study was to evaluate the safety and feasibility of bivalirudin during LAAO.

Methods: This retrospective, observational study included 420 AF patients who were evaluated as being at high risk of stroke or bleeding and indicated for LAAO at our center between June 2018 and June 2019 (158 with bivalirudin and 262 with heparin). The primary outcome was the incidence of any bleeding within 48 hours of LAAO. Secondary outcomes were major adverse cardiac events (MACE) between 48 hours and 60 days post-procedure and overall bleeding events during follow up.

Results: No significant difference was observed between bivalirudin and heparin for major periprocedural bleeding (1.27\% for bivalirudin vs. 2.29\% for heparin, $\mathrm{P}=0.716)$ or minor bleeding $(1.27 \%$ vs. $1.15 \%, \mathrm{P}>0.9)$. At 48 hours post-procedure, strokes occurred at a rate of $0.63 \%$ in the bivalirudin group and $1.15 \%$ in the heparin group $(\mathrm{P}>0.9)$, and one case treated with bivalirudin developed systemic embolization. At 60 days, the rates of MACE (1.90\% vs. 2.29\%, P>0.9), a device-related thrombus (DRT) $(1.27 \%$ vs. $1.52 \%, \mathrm{P}>0.9)$, and overall bleeding events $(5.06 \%$ vs. $4.96 \%, \mathrm{P}=0.963)$ were comparable between the 2 cohorts. Upon Kaplan-Meier survival analysis, early safety during the 60 -day follow-up was $93.67 \%$ in the bivalirudin group and $91.60 \%$ in the heparin group $(\mathrm{P}=0.570)$.

Conclusions: Bivalirudin has a comparable safety and efficacy profile to heparin as an intraprocedural anticoagulant, but currently, it should still be reserved for patients in which heparin is contraindicated.

Keywords: Left atrial appendage occlusion (LAAO); atrial fibrillation (AF); bivalirudin; heparin

Submitted Jun 17, 2020. Accepted for publication Jan 17, 2021.

doi: $10.21037 / \mathrm{atm}-20-4755$

View this article at: http://dx.doi.org/10.21037/atm-20-4755

\section{Introduction}

In recent years, percutaneous left atrial appendage occlusion (LAAO) has emerged as a reliable strategy for stroke prevention in atrial fibrillation (AF) patients at high risk of embolization and hemorrhage (1). However, with the cumulative experience gained from the rapid expansion of LAAO treatment, researchers have confirmed 
that LAAO is not inferior to anticoagulant therapy in terms of overall bleeding rates due to procedure-related bleeding incidence in the device group (2,3). Moreover, thrombotic microemboli to the brain or elsewhere at the time of implantation has also been documented as a specific periprocedural risk (4).

In randomized trials and daily practice, heparin has been acknowledged as the current standard empiric anticoagulant during LAAO and can be well reversed by protamine (1). On the other hand, marked interindividual variability can be observed regarding heparin's biological efficacy, and monitoring is required throughout the procedure to prevent bleeding events. Also, heparin-induced thrombocytopenia or allergy is uncommon but can be clinically disastrous (5). Patients with strong indications for LAAO are highly likely to be evaluated at high risk of stroke and bleeding at the baseline, and it has been reported that in Asian countries, hepatitis carrier status and liver cirrhosis are commonly encountered in AF patients $(6,7)$. Hence, these complications are not only a thorny pre-procedural problem-one that could leave the patient inoperablebut also significantly further increase their incidence of hemorrhagic and thrombotic events after implantation (1). Furthermore, heparin use is also associated with an increase in platelet reactivity. Hence, based on precautious manipulation, whether or not there is another option for periprocedural anticoagulation therapy in addition to heparin during LAAO has gradually emerged as a concern.

Bivalirudin is an irreversible and direct thrombin inhibitor with a half-life of approximately 25 minutes. The pharmacokinetics, pharmacodynamics, and anticoagulant effects of bivalirudin are more predictable as they do not bind to plasma proteins. It exerts a pharmacological effect upon the combination with antithrombin III, and it is not neutralized by platelet factor 4 antibodies (8). Notably, bivalirudin also eliminates the risk of heparin-induced thrombocytopenia $(1,2)$ due to it not binding with platelet factor 4 . In percutaneous coronary artery interventions (PCI) and transcatheter aortic valve replacements (TAVR), multiple studies have provided evidence on the comparable efficacy of heparin and bivalirudin as intraprocedural anticoagulants $(9,10)$. A trend towards the lower incidence of access or non-access hemorrhagic complications has also been indicated in several literature pieces, but this is not universally acknowledged $(11,12)$. This has led to an interest in investigating whether the use of bivalirudin can also provide another option of intraprocedural pharmacology in LAAO.
Therefore, the purpose of this study was to evaluate the safety and efficacy of periprocedural bivalirudin therapy during LAAO vs. weight-adjusted heparin among high-risk AF patients undergoing LAAO in a real-world setting.

We present the following article in accordance with the STROBE reporting checklist (available at http://dx.doi. org/10.21037/atm-20-4755).

\section{Methods}

\section{Study design and participants}

This retrospective, observational study was conducted in Zhongshan Hospital, Fudan University. From June 2018 to June 2019, a sample of consecutive patients $(n=420)$ referred for percutaneous LAAO with $\mathrm{AF}$ at high risk of stroke or bleeding (CHA2DS2 VASC $\geq 2$ or HAS-BLED $\geq 3$ ) at our institution were retrospectively enrolled in this research. A total of 158 participants were administered with bivalirudin, and heparin was used in 262 patients based on individual patient choice and the surgeon's discretion. Patients were excluded if LAAO was applied as an adjunctive part of other cardiac interventions. Exclusion criteria also included those patients that had been treated with intravenous heparin before LAA closure.

The study was conducted in accordance with the Declaration of Helsinki (as revised in 2013). Our study was approved by the Institutional Review Board of Zhongshan Hospital, Fudan University, Shanghai, China (B2020-042) and individual consent for this retrospective analysis was waived.

\section{Procedure}

Participants with a therapeutic international normalized ratio (INR) were continued with warfarin or new oral anticoagulants (NOACs) from admission to the hospital, and this anticoagulant therapy was withdrawn on the day of surgery to reach INR $<2.0$, following our institutional norms.

The LAAO procedure was performed in line with the description provided in previous literature (1). After a successful transseptal puncture, patients in the bivalirudin group received an initial bolus injection of $0.75 \mathrm{mg} / \mathrm{kg}$ followed by an intravenous infusion of $1.75 \mathrm{mg} / \mathrm{kg} / \mathrm{h}$ during LAAO. For patients with renal insufficiency (an estimated glomerular filtration rate of $30-59 \mathrm{~mL} / \mathrm{min}$ ), the infusion rate was decreased to $1.4 \mathrm{mg} / \mathrm{kg} / \mathrm{h}$. A 
supplemental bivalirudin infusion of $0.25 \mathrm{mg} / \mathrm{kg} / \mathrm{h}$ was maintained in this group until 4 hours after the procedure. Heparin participants were recommended a dosage of 70 to $100 \mathrm{IU} / \mathrm{kg}$, and an additional bolus was administered to achieve a target activated clotting time (ACT) of over 250 seconds. The surgeon used their clinical discretion to determine whether reversal with protamine needed to be administered at the end of the operation.

All participants were monitored with transthoracic echocardiograms (TTE) for at least 2 days before discharge. A planned 60-day course of oral anticoagulants (OACs) was recommended, and routine clinic visits with transesophageal echocardiography (TEE) examinations at a 60-day follow up was required of every participant to determine whether anticoagulation should be prolonged [if device-related thrombus (DRT) was detected].

\section{Definitions and outcomes}

The primary outcome of interest was the incidence of any bleeding events within 48 hours following procedure based on the Bleeding Academic Research Consortium (BARC) bleeding criteria, whose definition has been described in detail in previous literature (13). Secondary outcomes of our study included the following: (I) the rate of major adverse cardiovascular events (MACE) (death, ischemic stroke, and systemic embolization) within 48 hours of LAAO, (II) the rate of bleeding events, MACE and DRT at the 60-day follow up, and (III) early safety, defined as patients undergoing LAAO without evidence of a composite endpoint of major bleeding, MACE, DRT, or acquired thrombocytopenia during the 60-day follow up period.

\section{Statistical analysis}

Continuous variables were presented as means \pm standard deviations (SD) and compared using the Student's $t$-test. Categorical variables were expressed as numbers (in the form of percentages) and compared by Pearson's $\chi^{2}$ test or Fisher's exact test. Time-to-event was presented via Kaplan-Meier curves and compared using the log-rank test. Cox regression analysis was used to calculate the hazard ratios (HRs) and the relative risks were described with a $95 \%$ confidence interval (CI). A 2 -sided $\mathrm{P}$ value $<0.05$ was considered statistically significant. All analyses were performed using the software SPSS version 26.0 (SPSS Inc,. Chicago, IL, USA).

\section{Results}

\section{Baseline characteristics}

A total of 420 eligible patients (mean age $68.99 \pm 8.33$ years, male $63.81 \%$ ) were enrolled in our study, of whom 158 $(37.62 \%)$ were treated with bivalirudin during LAAO, and $262(62.38 \%)$ were treated with heparin. The participants' baseline characteristics, including gender, AF type, previous medical history, and proportion of anticoagulation therapy before admission, were well-matched between groups (Table 1). Participants presenting with prior stroke/transient ischemic attack (TIA) and hemorrhagic events were equally frequent between groups, and there were no significant differences between the bivalirudin and heparin groups regarding CHA2DS2-VASC and HAS-BLED scores.

\section{Procedural characteristics and post-procedural medication}

Procedural and treatment data are shown in Table 2. The LAAO procedure was attempted in 420 participants and was successfully performed in 419 participants. Only 1 case failed due to complex LAA morphology, and OACs were suggested as the ongoing treatment. No differences were found regarding device type, total operation time, and contrast volume during LAAO between the bivalirudin and heparin groups. Echocardiographic findings were also similar between the 2 groups, and no serious residual leaks after LAA closure were detected on color Doppler TEE in our study population. Furthermore, there were no significant differences regarding the discharge of anticoagulation therapy between the 2 groups.

\section{Clinical outcomes}

The periprocedural and 60-day outcomes are shown in Table 3. Generally, the primary and secondary outcomes were comparable between the 2 cohorts in our study population.

Life-threatening bleeding was not reported in our study, and no significant differences were observed between the bivalirudin group and the heparin group regarding major bleeding $(1.27 \%$ vs. $2.29 \%, \mathrm{P}=0.716)$ and minor bleeding ( $1.27 \%$ vs. $1.15 \%, \mathrm{P}>0.9)$ within 48 hours. Among them, serious pericardial effusion was reported in 2 participants in the bivalirudin group and in 4 participants in the heparin group, which accounted for nearly two-thirds of the major bleeding events $(60.00 \%)$ and half of the overall bleeding 
Table 1 Baseline characteristics of the study population

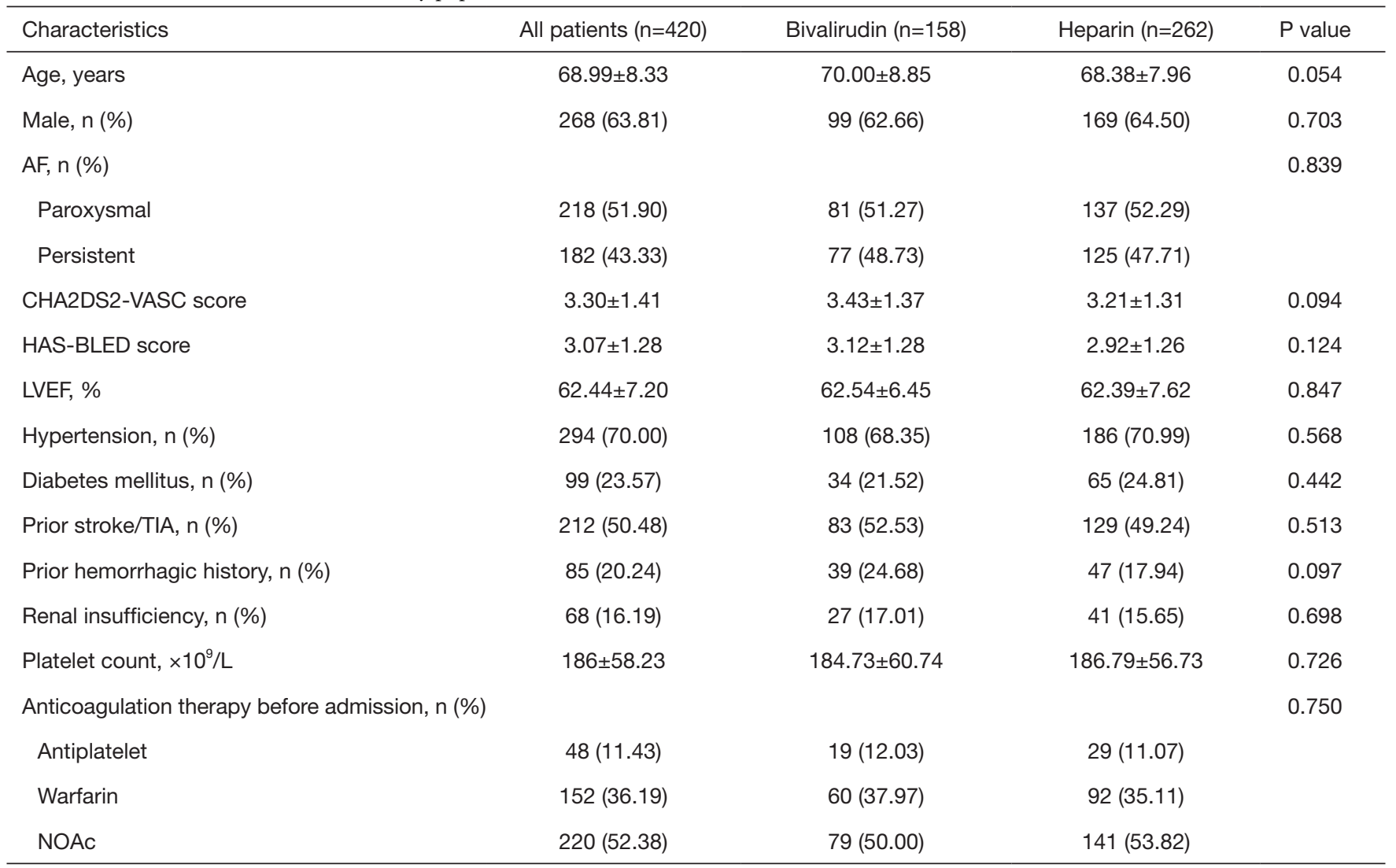

AF, atrial fibrillation; LVEF, left ventricular ejection fraction; TIA, transient ischemic attack; NOAc, new oral anticoagulation.

Table 2 Procedural characteristics between patients receiving bivalirudin and heparin

\begin{tabular}{|c|c|c|c|c|}
\hline Characteristics & All patients $(n=420)$ & Bivalirudin $(n=158)$ & Heparin $(n=262)$ & $P$ value \\
\hline Device type, n (\%) & & & & 0.197 \\
\hline WATCHMAN & $344(81.90)$ & $124(78.48)$ & $220(83.97)$ & \\
\hline LAMBRE & $75(17.86)$ & $33(20.89)$ & $42(16.03)$ & \\
\hline Volume of contrast, $\mathrm{mL}$ & $87.18 \pm 17.28$ & $86.76 \pm 18.84$ & $87.43 \pm 16.30$ & 0.710 \\
\hline Post-procedural antithrombotic therapy, n (\%) & & & & 0.268 \\
\hline DAPT & $131(31.19)$ & $44(27.85)$ & $87(33.21)$ & \\
\hline NOAC & $338(80.48)$ & $113(71.52)$ & $175(66.79)$ & \\
\hline
\end{tabular}

DAPT, dual antiplatelet therapy; NOAc, new oral anticoagulation. 
Table 3 Clinical outcomes between bivalirudin and heparin group

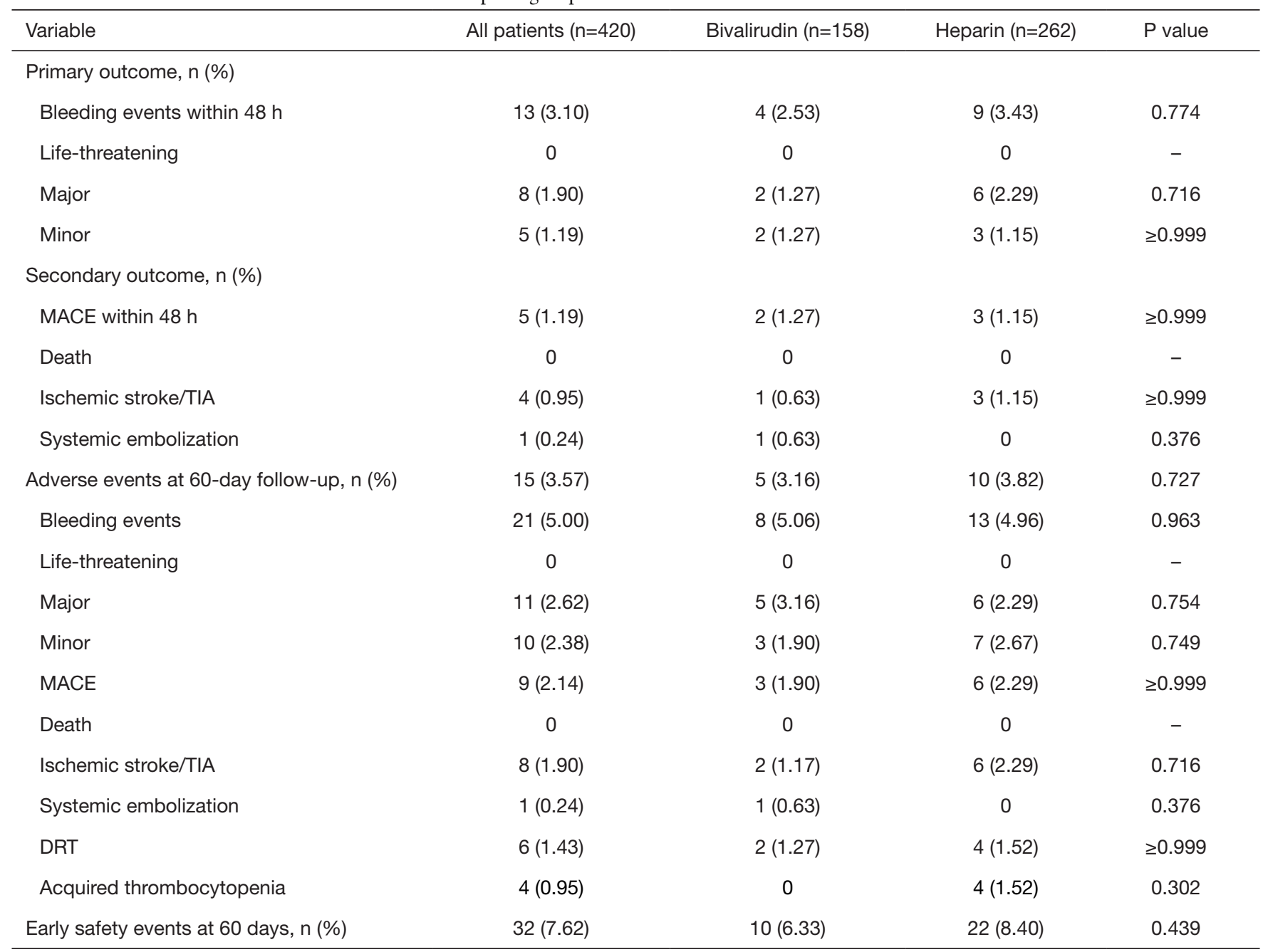

TIA, transient ischemic attack; DRT, device-related thrombus; MACE, major adverse cardiac events.

events (46.15\%) (Figure 1). Successful pericardiocentesis was performed in all of the six participants without further need for surgical repair. During hospitalization, ischemic stroke occurred in $0.63 \%$ of participants treated with bivalirudin vs. $1.15 \%$ of those treated with heparin $(\mathrm{P}>0.9)$. Also, another participant in the bivalirudin group developed a left subclavian artery embolism.

None of the participants were lost during followup at the 2-month mark, and neither death nor systemic embolization was documented. Participants treated with bivalirudin showed a $5.06 \%$ rate of overall bleeding, similar to the $4.96 \%$ rate in the heparin cohort $(\mathrm{P}=0.963)$. The MACE rates at the 60 -day follow up were $1.90 \%$ for bivalirudin and $2.29 \%$ for heparin $(\mathrm{P}>0.9)$. The incidence of DRT based on TEE readings taken at the 60-day follow- up was $1.27 \%$ in the bivalirudin group vs. $1.52 \%$ in the heparin group $(\mathrm{P}>0.9)$. Acquired thrombocytopenia was only observed in participants treated with heparin during hospitalization $(n=4)$, but the difference between the 2 groups was not statistically significant $(\mathrm{P}=0.302)$.

In summary, $92.38 \%$ of all cases had met our study's early safety endpoint at day 60, and the occurrence of the early safety endpoint was similar between the bivalirudin group $(93.67 \%)$ and the heparin group $(91.60 \%)$, as indicated in the Kaplan-Meier survival curve (log-rank 0.624, $\mathrm{P}=0.430$ ) (Figure 2). Upon Cox proportional hazard analysis after having adjusted for age and prior hemorrhagic history, the use of bivalirudin was still not a predictor for the early safety endpoint (HRs: 1.452; 95\%, CI: 0.690 to 3.099, $\mathrm{P}=0.335$ ).

Furthermore, subgroup analysis of the bleeding and 
Page 6 of 10

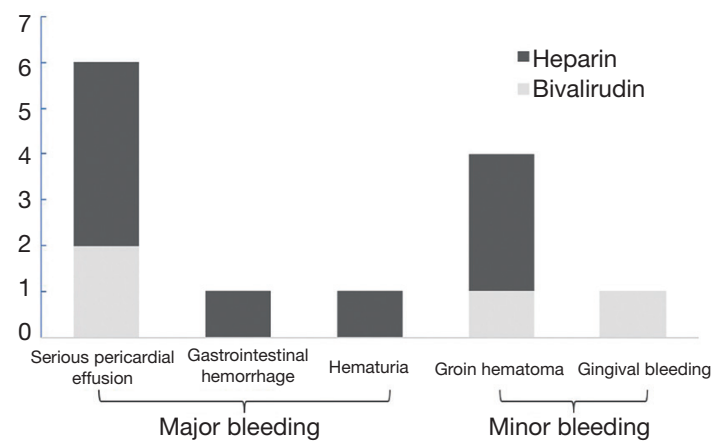

Figure 1 Types and frequencies of bleeding events within 48 hours after the LAAO procedure. LAAO, left atrial appendage occlusion.

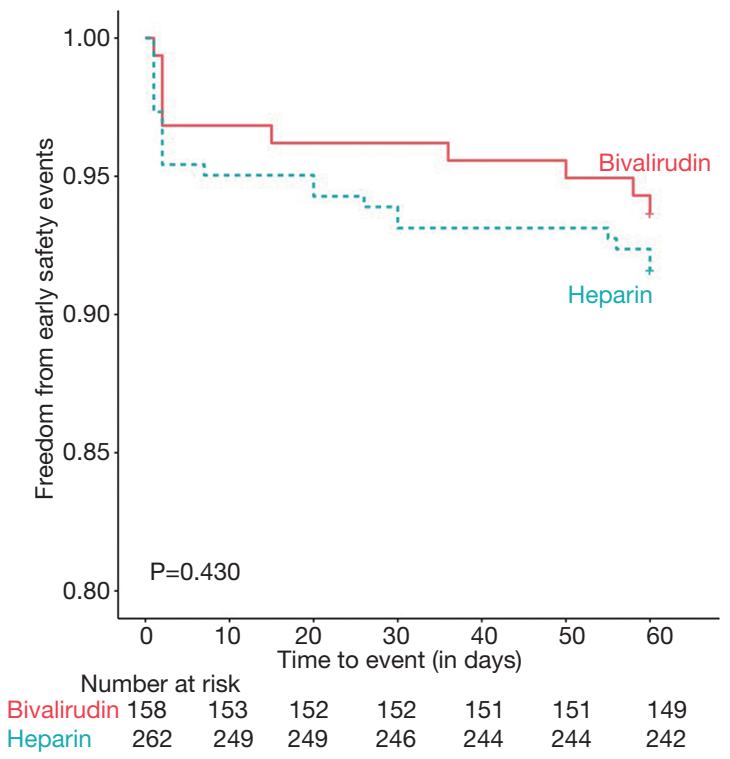

Figure 2 A cumulative Kaplan-Meier analysis showing freedom from early safety events within 60 days, stratified by treatment.

thromboembolic endpoint based on clinical characteristicssuch as age dichotomized at 70 years old, gender, renal insufficiency, or OAC post-procedure-revealed no clear significant differences between the 2 anticoagulation treatments during LAAO (Figure 3).

\section{Discussion}

This was the first clinical study to examine bivalirudin utilization in AF patients with a high risk of stroke or bleeding while undergoing LAAO. According to this relatively large observational study comparing bivalirudin and heparin as intraprocedural anticoagulants in LAA closure, we detected no differences in terms of both early bleeding and overall bleeding rates. Moreover, there were no significant differences between bivalirudin and heparin regarding MACE occurrence at 48 hours and 2 months post-procedure or concerning 2 -month early safety outcomes.

Procedure-related events occurring at the time of LAA closure (e.g., pericardial effusion or groin hematoma) have been demonstrated as the major drivers of bleeding during implantation, with the capacity to reduce the feasibility and safety of the procedure significantly. As a stroke prevention procedure for AF patients, LAAO lacks an immediately obvious benefit, and it is vital to prevent adverse procedural events to determine the overall risk-benefit ratio of the LAAO procedure.

In our study, the participants were exclusively AF patients at high risk for stroke or bleeding. The LAAO was performed at our center by experienced surgeons using Watchman devices (Boston Scientific, Marlborough, MA, USA). The overall procedural success rate of our study was $99.76 \%$, and intraprocedural imaging was used to ensure that the position, anchor, size, and seal (PASS) criteria were uniformly met. However, our findings in the heparin group still revealed a bleeding rate of $3.43 \%$ during the perioperative period, comparable to the $4.9-5.4 \%$ rates documented in previous registered studies on LAAO using heparin $(2,3)$.

Bivalirudin is a synthetic bivalent direct thrombin inhibitor with a short half-life that works independently of plasma proteins. The drug enables predictable pharmacokinetics and anticoagulant effects based on its dose-response curve and does not necessitate frequent laboratory monitoring (8). In our study, although no significant differences were observed at the primary endpoint of early bleeding, we noted that all bleeding and major bleeding rates were numerically lower in participants treated with bivalirudin. These results are similar to those achieved by bivalirudin in other studies of structural heart diseases, such as BRAVO 3 (14), a multicenter randomized controlled trial of bivalirudin versus heparin in patients undergoing transfemoral TAVR, which demonstrated comparable bleeding outcomes before discharge between the 2 anticoagulation treatments. We assumed that the limited sample size might have contributed to the lack of significant differences in our study population.

On the other hand, the current bivalirudin analyses for endovascular utilization focus almost entirely on the transarterial pathway $(15,16)$. Given the $14 \mathrm{~F}$ delivery sheath 
A

$\begin{array}{lr}\begin{array}{l}\text { Subgroups } \\ \text { Age }\end{array} & \text { Odds ratio }[95 \% \mathrm{Cl}] \\ >=70 & 0.964[0.215,4.416] \\ <70 & 2.537[0.291,22.151] \\ \text { Gender } & \\ \text { Male } & 0.982[0.229,4.199] \\ \text { Female } & 2.578[0.281,23.64] \\ \text { EGFR } & \\ >=60 & 1.633[0.426,6.269] \\ <60 & 0.591[0.035,9.853]\end{array}$

\section{B}

Subgroups

Age

$>=70$

$<70$

Gender

Male

Female

Post Procedure

DAPT

NOAC

EGFR

$>=60$

$<60$
Bleeding within $\mathbf{4 8 h}$

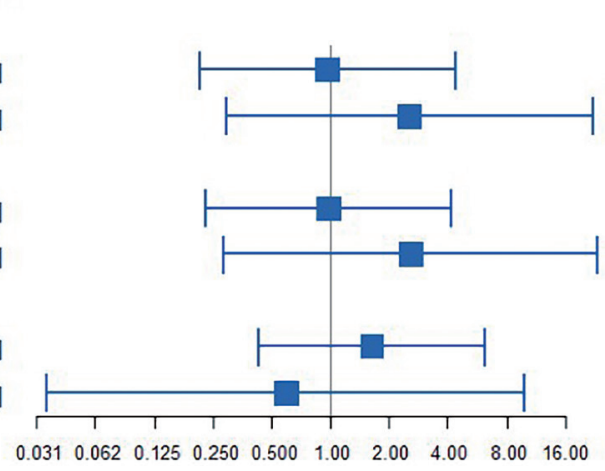

Bleeding within 60days

Odds ratio $[95 \% \mathrm{Cl}]$

$0.394[0.112,1.389]$

$4.708[0.584,37.936]$

$0.674[0.22,2.065]$

$1.943[0.379,9.964]$

$1.556[0.301,8.044]$

$0.75[0.245,2.292]$

$1.346[0.457,3.963]$

$0.372[0.058,2.384]$

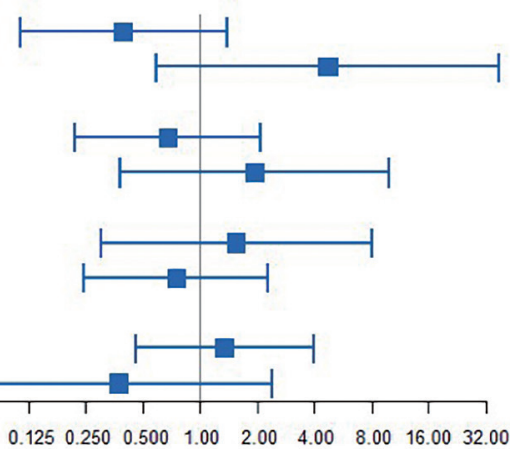

Adverse cardiac events within 60days

C

Subgroups

Age

$>=70$

$<70$

Gender

Male

Female

Post Procedure

DAPT

NOAC

EGFR

$>=60$

$<60$
Odds ratio $[95 \% \mathrm{Cl}]$

$0.9[0.235,3.453]$

$2.537[0.291,22.151]$

$1.185[0.29,4.848]$

$1.267[0.225,7.141]$

$0.494[0.067,3.631]$

$1.772[0.46,6.826]$

$1.058[0.304,3.687]$

$1.857[0.183,18.811]$

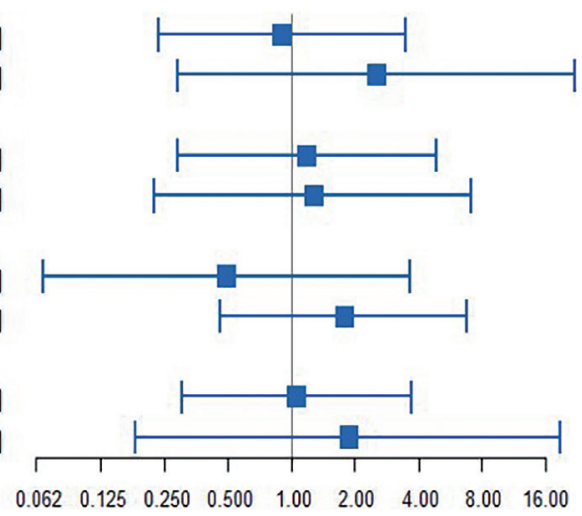

Figure 3 Subgroup stratification for bleeding and major adverse events between the bivalirudin and heparin groups. (A) Subgroup stratification for overall bleeding events within 48 hours of operation. (B) Subgroup stratification for overall bleeding events within 60 days of operation. (C) Subgroup stratification for thromboembolization (including stroke, systemic embolism, and device-related thrombus) within 60 days of operation. 
via a transvenous approach for Watchman implantation, this different operative approach might have diminished the significance. Regarding individual factors, serious pericardial effusion remained the dominant cause for major bleeding, and groin bleeding at the access site was the main reason for minor bleeding in both cohorts, similar to previously reported results (17). Despite the lack of a bivalirudin antidote, as with protamine reversal in heparin, no deaths occurred upon emergency intervention in any group. It is also remarkable that access site bleeding was only detected in our heparin cohort. Our results support a previous meta-analysis suggesting that bivalirudin may correlate to a comparable mortality benefit while preserving much of the groin hematoma reduction of transfemoral intervention (18).

Moreover, our study revealed that a surprisingly high proportion of 60-day hemorrhagic events were bleeding that occurred during hospitalization, with $50 \%$ in the bivalirudin group and $69 \%$ in the heparin group. This result further confirmed that the prerequisite for LAAO is the technical success of implantation. Further trials should focus on the relationship between the anticoagulants administered during implantation, and periprocedural complications might offer a new angle for finding the optimal prognostic cut-point for LAA closure.

In addition to bleeding events, the occurrence of thrombosis reflected by stroke and vascular complications during and after LAAO might also be associated with intraprocedural anticoagulation. Our study found that the rates of stroke $(0.63 \%$ for bivalirudin and $1.15 \%$ for heparin) and systemic embolism ( $0.63 \%$ for bivalirudin) were extremely low and not different between the groups; this similarity continued during follow up. At 60 days, there were no significant differences in the DRT rates between participants treated with heparin and bivalirudin, which was consistent with the indication that the LAA device's progressive endothelialization is determined mainly by the duration and agents of oral pharmacotherapy (19). Notably, other complications, such as acquired thrombocytopenia, were only identified in our study's heparin group.

Indeed, to directly inhibiting fluid-phase and fibrinbound thrombin, bivalirudin blocks protease-activated receptor 4 of platelets, eliminating the risk of heparininduced thrombocytopenia (20). Our study suggested bivalirudin's feasibility in this particular population, as observed in other studies $(21,22)$. In this context, we extended the corrected dosage of bivalirudin to the recommended dose for TAVR instead of that for coronary intervention $(9,14)$. Since a linear relationship has been reported between eliminating bivalirudin and renal function, our specifically decreased bivalirudin dosage showed sufficient prevention against ischemic events without increasing the bleeding risk compared to heparin. This, however, still requires testing in further trials.

To further establish an integral evaluation of bivalirudin in terms of safety and efficacy, a combined endpoint was determined as a counterbalance between the drug's intraprocedural anticoagulation benefits during the prevention of thromboembolism and its risk of side effects such as bleeding. The satisfactory early safety outcome of our study provided substantial preliminary evidence in support of bivalirudin as a favorable option for anticoagulation in addition to heparin for $\mathrm{AF}$ patients undergoing LAAO.

Regarding the effects of participants' baseline characteristics on clinical outcomes, we performed further analyses of a subgroup in which bivalirudin displayed a consistently similar risk of bleeding and thromboembolism regardless of age, gender, and renal function. Significantly, it has been announced that the variation of OACs postprocedure is an underlying triggering factor for bleeding $(23,24)$. Our results during follow-up demonstrated that the heparin and bivalirudin approaches produce comparable composite endpoints for hemorrhagic and stroke events, regardless of whether dual antiplatelet therapy (DAPT) or non-vitamin $\mathrm{K}$ antagonist oral anticoagulants (NOAc) was administered. Our study's unexpected finding was that all of the bleeding events that occurred within 48 hours in participants for which DAPT was administered occurred within the heparin group. Further research may be warranted to explore the plausible biological mechanism at work here.

\section{Limitations}

Our study was retrospective, containing a prospective analysis that sought to evaluate the clinical outcomes involved in the use of bivalirudin in LAAO among a realworld population. Due to this study's non-randomized nature, along with its limited sample size from a singlecenter, we cannot exclude the possibility of selection bias in our study groups. Some baseline clinical variables of the 2 groups were still borderline significant; thus, the differences between these 2 strategies could be minimized by these confounding effects. Moreover, most of the incidence rates of efficacy and safety events were lower than 
0.05 . This particularly low rate of events in our study has little statistical power and might have led to false-negative findings. In the future, larger, randomized, prospective, controlled, multicenter investigations on bivalirudin use compared to heparin in LAAO are warranted to explore further whether or not the clinically negligible difference between the clinical outcomes in our study was due to chance.

\section{Conclusions}

In summary, our study results suggest that the periprocedural use of bivalirudin in LAAO among AF patients at a high risk of stroke or bleeding is associated with positive outcomes, including a favorable bleeding risk and low incidence of major adverse cardiac events. Considering bivalirudin's higher cost and the insignificant advantage observed in our study, we propose that, at present, bivalirudin should continue to be intensively applied as a peri-LAAO anticoagulant in clinical practice.

\section{Acknowledgments}

Funding: This work was supported by the National Natural Science Foundation of China (81771837) and the Science and Technology Commission of Shanghai Municipality (17411962400).

\section{Footnote}

Reporting Checklist: The authors have completed the STROBE reporting checklist. Available at http://dx.doi. org/10.21037/atm-20-4755

Data Sharing Statement: Available at http://dx.doi. org/10.21037/atm-20-4755

Conflicts of Interest: All authors have completed the ICMJE uniform disclosure form (available at http://dx.doi. org/10.21037/atm-20-4755). The authors have no conflicts of interest to declare.

Ethics Statement: The authors are accountable for all aspects of the work in ensuring that questions related to the accuracy or integrity of any part of the work are appropriately investigated and resolved. The study was conducted in accordance with the Declaration of Helsinki (as revised in 2013). The study was approved by the
Institutional Review Board of Zhongshan Hospital, Fudan University(B2020-042), and individual consent for this retrospective analysis was waived.

Open Access Statement: This is an Open Access article distributed in accordance with the Creative Commons Attribution-NonCommercial-NoDerivs 4.0 International License (CC BY-NC-ND 4.0), which permits the noncommercial replication and distribution of the article with the strict proviso that no changes or edits are made and the original work is properly cited (including links to both the formal publication through the relevant DOI and the license). See: https://creativecommons.org/licenses/by-nc-nd/4.0/.

\section{References}

1. Glikson M, Wolff R, Hindricks G, et al. EHRA/EAPCI expert consensus statement on catheter-based left atrial appendage occlusion - an update. Europace 2019;euz258.

2. Price MJ, Reddy VY, Valderrabano M, et al. Bleeding Outcomes After Left Atrial Appendage Closure Compared With Long-Term Warfarin: A Pooled, Patient-Level Analysis of the WATCHMAN Randomized Trial Experience. JACC Cardiovasc Interv 2015;8:1925-32.

3. Holmes DR, Doshi SK, Kar S, et al. Left Atrial Appendage Closure as an Alternative to Warfarin for Stroke Prevention in Atrial Fibrillation. J Am Coll Cardiol 2015;65:2614-23.

4. Gloekler S, Saw J, Koskinas KC, et al. Left atrial appendage closure for prevention of death, stroke, and bleeding in patients with nonvalvular atrial fibrillation. Int J Cardiol 2017;249:234-46.

5. Salter BS, Weiner MM, Trinh MA, et al. Heparin-Induced Thrombocytopenia: A Comprehensive Clinical Review. J Am Coll Cardiol 2016;67:2519-32.

6. Lee H, Choi EK, Rhee TM, et al. Cirrhosis is a risk factor for atrial fibrillation: A nationwide, population-based study. Liver Int 2017;37:1660-7.

7. Mwalitsa JP, Maimone S, Filomia R, et al. Atrial fibrillation in patients with cirrhosis. Liver Int 2016;36:395-400.

8. Capodanno D, De Caterina R. Bivalirudin for acute coronary syndromes: premises, promises and doubts. Thromb Haemost 2015;113:698-707.

9. Lange $\mathrm{P}$, Greif $\mathrm{M}$, Bongiovanni $\mathrm{D}$, et al. Bivalirudin vs Heparin in Patients Who Undergo Transcatheter Aortic Valve Implantation. Can J Cardiol 2015;31:998-1003.

10. Mavrakanas TA, Chatzizisis YS. Bivalirudin in stable angina and acute coronary syndromes. Pharmacol Ther 
2015;152:1-10.

11. Nührenberg TG, Hochholzer W, Mashayekhi K, et al. Efficacy and safety of bivalirudin for percutaneous coronary intervention in acute coronary syndromes: a meta-analysis of randomized-controlled trials. Clin Res Cardiol 2018;107:807-15.

12. Gargiulo G, Carrara G, Frigoli E, et al. Bivalirudin or Heparin in Patients Undergoing Invasive Management of Acute Coronary Syndromes. J Am Coll Cardiol 2018;71:1231-42.

13. Tzikas A, Holmes DR Jr, Gafoor S, et al. Percutaneous left atrial appendage occlusion: the Munich consensus document on definitions, endpoints, and data collection requirements for clinical studies. Europace 2017;19:4-15.

14. Dangas GD, Lefèvre T, Kupatt C, et al. Bivalirudin Versus Heparin Anticoagulation in Transcatheter Aortic Valve Replacement: The Randomized BRAVO-3 Trial. J Am Coll Cardiol 2015;66:2860-8.

15. Lincoff AM, Kleiman NS, Kereiakes DJ, et al. Long-term efficacy of bivalirudin and provisional glycoprotein IIb/ IIIa blockade vs heparin and planned glycoprotein IIb/IIIa blockade during percutaneous coronary revascularization: REPLACE-2 randomized trial. JAMA 2004;292:696-703.

16. Kastrati A, Neumann FJ, Mehilli J, et al. Bivalirudin versus unfractionated heparin during percutaneous coronary intervention. N Engl J Med 2008;359:688-96.

17. Thakkar J, Vasdeki D, Tzikas A, et al. Incidence, Prevention, and Management of Periprocedural Complications of Left Atrial Appendage Occlusion. Interv Cardiol Clin 2018;7:243-52.

18. Shah R, Rogers KC, Matin K, et al. An updated

Cite this article as: Zhang $\mathrm{X}$, Jin Q, Kong D, Pan C, Zhang X, Zhou D, Shen Z, Zhou D, Ge J. Clinical outcomes of bivalirudin versus heparin in atrial fibrillation patients undergoing percutaneous left atrial appendage occlusion. Ann Transl Med 2021;9(8):629. doi: 10.21037/atm-20-4755 comprehensive meta-analysis of bivalirudin vs heparin use in primary percutaneous coronary intervention. Am Heart J 2016;171:14-24.

19. Saw J, Nielsen-Kudsk JE, Bergmann M, et al. Antithrombotic Therapy and Device-Related Thrombosis Following Endovascular Left Atrial Appendage Closure. JACC Cardiovasc Interv 2019;12:1067-76.

20. Aggarwal A, Sobel BE, Schneider DJ. Decreased platelet reactivity in blood anticoagulated with bivalirudin or enoxaparin compared with unfractionated heparin: implications for coronary intervention. J Thromb Thrombolysis 2002;13:161-5.

21. Joseph J, Rabbolini D, Enjeti AK, et al. Diagnosis and management of heparin-induced thrombocytopenia: a consensus statement from the Thrombosis and Haemostasis Society of Australia and New Zealand HIT Writing Group. Med J Aust 2019;210:509-16.

22. Joseph L, Casanegra AI, Dhariwal M, et al. Bivalirudin for the treatment of patients with confirmed or suspected heparin-induced thrombocytopenia. J Thromb Haemost 2014;12:1044-53.

23. Bergmann MW, Betts TR, Sievert H, et al. Safety and efficacy of early anticoagulation drug regimens after WATCHMAN left atrial appendage closure: three-month data from the EWOLUTION prospective, multicentre, monitored international WATCHMAN LAA closure registry. EuroIntervention 2017;13:877-84.

24. Reddy VY, Gibson DN, Kar S, et al. Post-Approval U.S. Experience With Left Atrial Appendage Closure for Stroke Prevention in Atrial Fibrillation. J Am Coll Cardiol 2017;69:253-61. 